Research Article

\title{
Optimization of Intelligent Logistics Supply Chain Management System Based on Wireless Sensor Network and RFID Technology
}

\author{
Changhong Pan $(\mathbb{D})$ and Meng Liu \\ School of Information and Business Management, Dalian Neusoft University of Information, Dalian, Liaoning 116023, China \\ Correspondence should be addressed to Changhong Pan; panchanghong@neusoft.edu.cn
}

Received 1 October 2021; Accepted 20 October 2021; Published 2 November 2021

Academic Editor: Guolong Shi

Copyright (c) 2021 Changhong Pan and Meng Liu. This is an open access article distributed under the Creative Commons Attribution License, which permits unrestricted use, distribution, and reproduction in any medium, provided the original work is properly cited.

\begin{abstract}
Based on the in-depth study of two technologies, wireless sensor network, and RFID technology, this paper proposes a corresponding solution to the urgent storage positioning problem and the safety of goods in transit in the logistics supply chain process. And on this basis, it broadens the whole logistics process of warehousing and distribution and designs a framework of intelligent logistics supply chain management system with wireless sensor network and RFID technology to realize the management process of logistics main business. For the problems of complicated hardware wiring, the excessive number of positioning nodes and low effective coverage rate of traditional RFID-based positioning methods in large-scale warehousing environment, the integrated RFID and wireless sensor network fusion technology constructs a new movable anchor node, divides the nodes in the traditional positioning process into fixed anchors and mobile anchors, proposes a secondary positioning method, improves positioning accuracy and effective coverage rate, and provides a new positioning method for large-scale storage environment. For the transportation of goods in transit, especially precious and dangerous goods, a six-axis acceleration sensor-based method for detecting the behavioral attitude of goods in transit is proposed. Time window slicing of cargo motion data stream is performed to reduce data processing and based on the statistical features of a large amount of experimental data, and feature vectors and classifiers are analyzed and designed to achieve behavioral attitude classification of goods in transit. The experiment proves that this method has high recognition accuracy and has certain practical application value. Finally, the whole storage and distribution logistics process is integrated, the framework of intelligent logistics supply chain management system combining WSN and RFID is built based on wireless sensor network software and hardware platform, and the detailed design of logistics supply chain management system business process is mainly carried out. Combined with the two key methods proposed in this paper, the information management process of traditional logistics business such as access, storage positioning and monitoring, and distribution monitoring and management is realized, and the management system is tested on this basis. The testing effect proves that this system has certain deployment reference significance and practical application value and to a certain extent can help improve the intelligence level of logistics supply chain management and achieve the expected goal of this study.
\end{abstract}

\section{Introduction}

The rationalization of information technology and equipment selection should first clarify the logistics supply chain information collection object. Logistics information includes two aspects, not only the information related to logistics activities but also the information related to other circulation activities [1]. Logistics information has the function of supporting and guaranteeing the storage and transportation operations and related logistics management activities and plays the role of integrating resources to improve efficiency. Logistics supply chain information also has the general characteristics of logistics information, and the management of logistics supply chain information should collect, organize, transmit, store, and utilize the relevant information of the whole process. Logistics supply chain informationization not only includes information management and transmission of warehousing and transportation but also includes support for various decision-making activities and management applications in the process, making full use of 
information technology, collecting, transmitting, and analyzing data flow to achieve the purpose of controlling and supervising it. The effective management of logistics supply chain information is a reliable means and way to achieve the goal of the logistics supply chain $[2,3]$. The object of logistics supply chain information management not only includes the basic information required for safety management and logistics operation but also includes the state information and characteristic information reflected by the basic information. This shows that the research on intelligent management and application of logistics supply chain should also focus on the information collection object in the process of logistics supply chain informatization and reasonably choose the informatization technology according to the information collection object, application scenario, and other factors to ensure the scientific and realistic nature of intelligent management and application [4].

The level of intelligent operation of the information system is limited by the scientific degree of the solution method of the related decision problems involved. Therefore, to bring into play the efficiency of intelligent operation of the information system, the information system should be constructed based on recognizing the essence of the related decision problems involved in the logistics supply chain, reshaping the solution and method of the decision problems, and guaranteeing the scientificity of the solution method of the related decision problems in the logistics supply chain. Therefore, it is necessary to carry out the optimization research of the relevant decision problems involved in the logistics supply chain, such as the storage decision or transportation decision in logistics supply chain management, to ensure the scientific nature of the intelligent management and application research of logistics supply chain based on RFID and WSN in the intelligent operation of logistics [5, 6]. This paper is based on RFID and WSN as the technical means and the classification of logistics supply chain management, designs the logistics supply chain management plan based on RFID and WSN, constructs the logical framework of the intelligent monitoring system, and integrates the intelligent decision support system into the logistics supply chain management system architecture to construct. The intelligent decision-making function module of the logistics supply chain intelligent monitoring system provides a theoretical framework for a complete logistics information system architecture [7]. Based on the status quo of logistics storage and transportation, analyze the characteristics of the logistics supply chain storage and transportation process, propose a process analysis method that meets the characteristics of the logistics supply chain storage and transportation process, and use this method to analyze the logistics supply chain storage and transportation process, aiming at the logistics supply chain storage existing deficiencies in the transportation process, find an effective way to optimize the logistics supply chain storage and transportation process, and provide a scientific method for the analysis and optimization of the logistics supply chain process [8].

By combining wireless sensor network and RFID technology with logistics supply chain management model, we can promote people to manage all aspects of the logistics supply chain dynamically, to improve the level of logistics supply chain management, reduce logistics costs and security monitoring costs, and thus provide consumers with high-quality and safe logistics products. The application of advanced logistics information technology such as wireless sensor networks and RFID technology to modern logistics supply chain management is the trend of future logistics development and is of great significance to promote the development of logistics supply chain management. This paper will combine the wireless sensor network and RFID technology with supply chain management and analyze the application of wireless sensor networks and RFID technology in logistics supply chain management and the design of the system based on the wireless sensor network and RFID technology in various aspects of supply chain management. The first chapter introduces the research background of the topic, the research purpose and significance, and the main content and technical route of this paper. Chapter 2 introduces the current research status of logistics supply chain management. Chapter 3 introduces the research of intelligent logistics supply chain management system based on wireless sensor network and RFID technology and introduces the composition of the system and its working principle and the application of wireless sensor network and RFID technology in logistics supply chain management, as well as the system design in each link based on wireless sensor network and RFID technology. Chapter 4 introduces the relevant result analysis, and the testing effect proves that this system has certain deployment reference significance and practical application value. Chapter 5 summarizes the full text and proposes that the future development of wireless sensor networks and RFID technology needs to overcome the conceptual barriers to the application of the new technology, label cost barriers, solve the existing technical problems, and get the complete application from the perspective of the whole supply chain to truly promote the future development of this technology.

\section{Related Work}

RFID has received wide attention in the field of logistics information with its excellent identification function, especially in some hot areas of logistics such as logistics monitoring. Lam and Ip et al. established an optimization model of logistics warehousing and distribution based on RFID technology mainly for forklift picking and moving problems, thus, improving the efficiency of warehousing and resource utilization, reducing logistics costs, and improving customer service quality [9]. In logistics sorting and distribution, the use of RFID systems, barcode identification, sensor networks, and mobile Internet technologies to reduce human labor in the handling and sorting of goods, the initial automation of goods in and out and picking, is the basis for the automation of logistics operations. The complementary characteristics of WSN technology and RFID technology make the combination of the two have great research prospects. Abdirad and Krishnan have fully described the advantages and disadvantages of both technologies and pointed out that the integration of the two technologies will be more 
efficient and reliable in engineering and scientific fields, especially in acquiring, processing, and distributing data in dynamic environments [10]. The application areas of wireless sensor networks in the logistics chain are analyzed by Sadeeq and Zeebaree [11]. The prospect of wireless sensor networks in logistics is analyzed mainly in the fields of warehouse environment monitoring, hazardous materials, and cold chain logistics management. WSN technology can collect various types of information and has the advantages of being massively configurable, unattended, long sensing distance, and low cost. Its disadvantage is that it only cares about collecting data, and the marking capability is not enough; in addition, there is some difficulty in low power consumption and high lifetime [12].

Ping et al. proposed a WSN-RFID colocation method by studying the positioning technology of passive UHF RFID and WSN network positioning technology and used the method to solve the problem of area positioning and precise positioning of storage vehicles on precise roads [13]. Lee et al. designed a supply chain management system with RFID and WSN integration, where RFID tags and identifies items and WSN performs the detection of the environment, and the design was simulated in the Petri network toolbox environment of Matlab to verify that the modular devices can spontaneously execute various commands and improve the system performance [14]. Attaran constructed a drug distribution IoT model based on the combination of RFID and WSN. The description model of state and process information in drug distribution and the storage model of distribution information were given mainly from the perspective of the data model, and the tracking process of drug distribution information was modeled by the Petri network toolbox of Matlab [15]. Process optimization is the process of reengineering processes by using process redesign techniques and selecting process redesign tools and process optimization methods. Process redesign techniques and tools are the core and important part of process reengineering $[16,17]$. The current study of warehouse management decision problems, usually in the case of warehouse storage state to determine the establishment of storage space allocation optimization model, did not transport process and arrival time uncertainty, resulting in changes in the warehouse storage state, as a warehouse space allocation model to consider the establishment of factors [18].

In the large-scale storage environment, storage positioning has been a key issue that restricts the efficiency of goods inventory and access, and the existing RFID-based positioning algorithms are mostly oriented to a small area of indoor positioning, for the shelf-based large-scale storage environment, the need to deploy too many RFID anchor nodes to achieve effective positioning signal coverage, wiring complex, hardware overhead is too large. WSN can make up for the lack of RFID networking and transmission capabilities, increasing the flexibility of node deployment $[19,20]$. In the logistics and distribution chain, the positioning system and manual inspection-based monitoring means cannot meet the demand for real-time monitoring of goods. However, such as the camera tracking video surveillance wiring complex and high requirements for light, sight distance con- ditions are not suitable for use in narrow transport containers. WSN's excellent wireless data transmission capabilities are ideal for the transmission of goods in transit monitoring data, and rich sensor interface can also provide a variety of monitoring conditions. Especially in gesture detection, acceleration sensors have been mostly seen in gesture recognition, motion recognition, and other gesture detection research. With the improvement of sensor technology, sixaxis acceleration sensors can provide more accurate acceleration and provide posture angle data compared to three-axis sensors. This study is aimed at solving the problems of warehouse positioning and safety monitoring of goods in transit in the logistics process and to providing a powerful solution for the information transformation of small and mediumsized logistics enterprises and self-built logistics, helping them to shorten the development cycle, reduce development costs, and improve development efficiency.

\section{Research on Intelligent Logistics Supply Chain Management System Based on Wireless Sensor Network and RFID Technology}

3.1. Pose Detection Method Based on Wireless Sensor Network. The six-axis acceleration sensor is a sensor that can sense acceleration and convert it into a usable output signal. It has the advantages of accurate measurement, stable performance, high reliability, and flexible use. The six-axis acceleration sensor is a sensor that converts nonelectricity changes into electricity. The changed originals are the parts of measuring and controlling instruments and equipment. With the objectives of maximizing the number of outgoing cargo space and minimizing the number of incoming cargo space, the decision optimization model of cargo space allocation is constructed by considering the constraints of storage taboo, type constraints, physical condition constraints, and quantity equilibrium, to coordinate the stage plan of cargo space allocation within a certain period. The decision optimization model $J: M$ of cargo space allocation is shown in the following equation.

$$
J: M=\lim _{H, K \longrightarrow \infty} \sum_{i=1}^{H} \sum_{j=1}^{K} m_{i j} .
$$

Equation (2) is for the number of cargo space allocation equilibrium relationship constraints, the number of inbound is equal to the sum of the number of assigned to all cargo space, and the number of outbound is equal to the sum of the number of sources of all cargo space.

$$
h_{i}=\lim _{K \longrightarrow \infty} \sum_{j=1}^{K} h_{i k}, i \subseteq[1, M] .
$$

Based on the existing research results for the selection of multimodal transport routes under selected time-varying conditions, the two optimization objectives are (1) the minimum risk caused by all transport in the transport network 
and (2) the minimum transport costs, including the cost of transporting sections and the cost of changing nodes. The social risk caused by all transportation in the transport network is shown in the following equation.

$$
M_{i}=\lim _{H, K, N \longrightarrow \infty} \sum_{i=1}^{H} \sum_{j=1}^{K} \sum_{k=1}^{N} A_{i} B_{i j}^{i k} C_{i j}^{i k} .
$$

The costs incurred for all transportation in the transportation road network are shown in the following equation.

$$
H_{i}=\lim _{K, N \longrightarrow \infty} \sum_{j=1}^{K} \sum_{k=1}^{N} A_{j} B_{i j}^{i k}+\lim _{K, N \longrightarrow \infty} \sum_{j=1}^{K} \sum_{k=1}^{N} A_{j} C_{i j}^{i k}, i \subseteq[1, M] .
$$

In the problem of pathway mode selection, we need to choose the next arrival node and the transportation mode. In this case, we first transform the original transport network. Moreover, the transport decisions are related not only to the population density of the next way segment and the transport cost but also to the cost of transport mode conversion at that node. Therefore, the heuristic information for this problem is defined as the following equation.

$$
f(i, j)=\frac{1}{X_{i j}^{i k} \otimes\left(A_{i j}^{k}+A_{i}^{k}\right)}+\frac{1}{X_{i j}^{i k} \otimes\left(B_{i j}^{k}+B_{i}^{k}\right)} .
$$

In the transport box, warehouse, vehicles, ships, aircraft, and other places of stay to install RFID systems, related monitoring sensing and environmental regulation and other facilities and equipment, transport, and combined storage business operation links for the unit configuration of frontend monitoring terminals, which transport links, transport tools installed satellite navigation system terminal. The front-end monitoring terminal can receive and record information including sensors, automatic identification terminals, satellite navigation terminals, relevant databases, environmental regulation equipment status, and video monitoring, screen and fuse useful information and process it, and store it in the database at the same time. Logisticsrelated personnel and institutions can access and query relevant information through the Internet route. The regulatory scheme mainly includes the content of security status monitoring, early warning and regulation equipment control, automatic identification of goods, vehicle positioning and navigation, and intelligent scheduling of logistics resources. The conceptual framework of logistics intelligent monitoring program based on RFID and WSN technology is shown in Figure 1.

In the process of storage, the front-end monitoring terminal uses automatic identification technology to automatically obtain basic and safety information, and the front-end monitoring terminal automatically generates storage restriction levels and rationalizes storage requirements according to the information of goods, provides storage management personnel with cargo space allocation plans and organiza- tion plans for loading and unloading facilities, provides storage management personnel with exit plans when leaving the warehouse, and provides reasonable transportation tool scheduling for transportation organization management. In the process of logistics transportation, transportation tools use satellite navigation system terminals to receive location information through satellite navigation system positioning services, and satellite navigation system terminals use their communication technology or other communication technology to assist and communicate with the front-end monitoring platform. This paper designs a cargo behavior and posture detection algorithm based on acceleration sensors. This method uses acceleration sensors to obtain data and combines the largest similarity of multifeature recognition. The algorithm divides the behavior and posture of the goods and uses the geographic information system to obtain the geographic location information of the goods in transit, so as to realize the intelligent active monitoring and tracking process of the goods in transit.

\subsection{Optimization of Positioning Algorithm Based on RFID} Technology. The accuracy of RFID positioning systems is influenced by the positioning method, but the choice of RFID tag type and the addition of a reference tag also play a decisive role in positioning accuracy. Various positioning solutions have their characteristics and advantages. In actual use, in addition to the consideration of positioning accuracy, the ease of arrangement of the system, expandability, and system cost are related to the choice of RFID positioning solution. RFID received signal strength is the indicator value given by the reader by measuring the signal strength received by itself. Different models have a significant impact on the ranking results, and this paper selects the logarithmic distance path loss model generally followed by the actual indoor environment for representation, as in equation (6). The unit of $H(d)$ in the formula is $\mathrm{dBm}$, which indicates the path loss when the distance between reader and label is $d ; d_{0}$ indicates the path loss when the near reference distance $d_{0} ; w$ is the path loss factor, which indicates the path loss rate of growth with distance, the value is environmentdependent, the path loss factor for different materials will be given in engineering or can be obtained by taking experimental measurements to fit the curve; $Z_{\beta}$ denotes a normal random variable with a standard deviation of $\beta$ and mean value of 0 .

$$
H(d)=Z_{\beta}+H\left(d_{0}\right)+w * \log _{2} \frac{d}{d_{0}} .
$$

The transmitted power of the signal is $H_{g}$ as in equation (7), where RFID $(d)$ is the received power of the signal at a distance of $d$.

$$
H(d)=H_{g}-\operatorname{RFID}(d) * w .
$$

This gives the distance $d$ versus the received signal strength RFID $(d)$ under this propagation model, as in the 


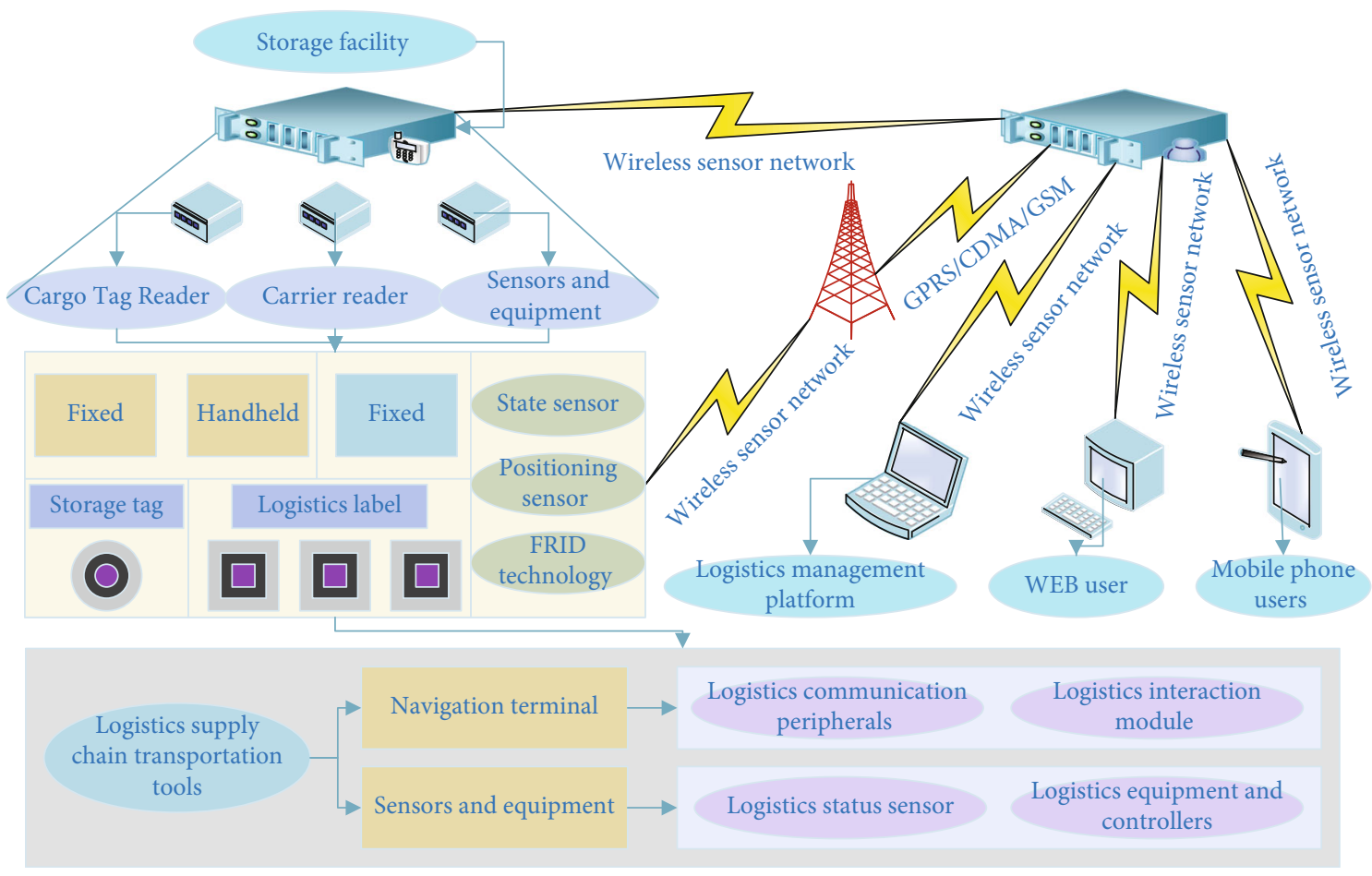

FIgURE 1: Conceptual framework of logistics intelligent monitoring solution based on RFID and WSN technology.

following equation.

$$
f(d)=f\left(d_{0}\right) * \sqrt[2]{\operatorname{RFID}\left(d_{0}\right) \oplus \operatorname{RFID}(d) \otimes Z_{\beta}}
$$

The trilateral measurement algorithm uses the coordinates of the nodes $A(m a, n a), B(m b, n b)$, and $C(m c, n c)$ at known locations and the corresponding estimated distances $d 1, d 2$, and $d 3$ calculated with the tag to be measured, and the geometric relationship can be used to obtain the plane position of the tag to be measured. Due to the influence of noise and other effects, the strength of the actual signal is often smaller than the estimated value of the position, increasing the derived estimated distance, so the measured radius is large, and the three circles do not intersect at a point but form an intersection. The position of the tag to be located is in this intersection. Therefore, in practical applications, the triangle center-of-mass localization algorithm is generally used, where the center-of-mass $M$ of the triangle with the intersection of the three circles as the vertex is used as the estimate of the actual point $O$. The coordinates of the intersection point of the three circles are calculated. In this case, the intersection point $D$ is calculated as shown in the following equation.

$$
\left\{\begin{array}{l}
f\left(d_{1}\right)=\sqrt{(m(d)-m(a))^{2}+(n(d)-n(a))^{2}}, \\
f\left(d_{2}\right)=\sqrt{(m(d)-m(b))^{2}+(n(d)-n(b))^{2}}, \\
f\left(d_{3}\right)=\sqrt{(m(d)-m(c))^{2}+(n(d)-n(c))^{2}} .
\end{array}\right.
$$

For the triangle mass center algorithm, the twodimensional plane under the three sides of the positioning still needs three known locations of the reader node and can be positioned range is only the intersection of three readers read and write range, that is, 3 degrees coverage area. If the location to be measured exceeds the intersection range, only in 1 degree or 2 degrees under the coverage area, it cannot be positioned. If it increases the read-write node one way or another, not only will it increase the hardware equipment overhead and the algorithm complexity, it also will bring communication interference and other problems. If it simply reduces the read-write node quantity, it will cause a larger scope of the uncovered area and affect the positioning accuracy. Due to the modern storage center's larger area, to interval arrangement shelves mainly placement scene, the traditional trilateral measurement or triangle mass center positioning algorithm cannot meet the positioning demand. Due to the influence of noise and other influences, the actual signal strength is often less than the estimated value of the location, which leads to an increase in the estimated distance derived. Therefore, the actual measured radius is too large. The three circles do not intersect at a point, but form an intersection. The position of the label to be located is in this intersection. Therefore, in practical applications, the triangle centroid positioning algorithm is generally used, and the centroid $M$ of the triangle with the intersection of the three circles as the vertex is used as the estimated value of the actual point $O$.

\subsection{Intelligent Logistics Supply Chain Management System} Design and Implementation. The wireless network as the center, integrated RFID readers, sensors, wireless repeaters, 
intelligent RFID terminals and data centers, and other integrated technologies to form a comprehensive network with wide coverage and strong sensing capabilities. In this network, the RFID reader first reads the RFID tag or RFID sensor tag data via RFID to identify a single product, while the sensor collects information about the surrounding environment. Then, the identity and environmental information of this single product will be sent to the wireless repeater, which will then be distributed to the corresponding subscribers in the wireless network via Wi-Fi protocol. As a subscriber of the information, the smart RFID terminal will filter, cluster, and encode the RFID information and sensing information after receiving it. For different application needs, the processed data will be stored directly in the remote data center or sent to other RFID service providers for further processing, or it can be integrated directly with the applications on the smart terminal through the business engine on the smart terminal for business processing. In this integrated networking scheme, the smart RFID terminal will assume more data processing and transmission functions as an edge server to prepare for the massive data processing of the IoT system. Therefore, the development of a flexible and scalable middleware on mobile devices will become the core technology of RFID and WSN integration.

Figure 2 shows the overall architecture of the intelligent logistics supply chain management system. According to the deployment method, this middleware can be divided into the mobile terminal part and the remote server part. The middleware of the mobile terminal includes the device access layer, data processing layer, business processing layer, and mobile application layer, while the remote server will further process the data according to ALE specification and connect to the enterprise's data center or public information platform. For example, in the production line of wine products, RFID information read by readers will be filtered and coded by mobile terminals and sent directly to the remote ALE server, which will update the back-end data center. Here, the mobile middleware will play the function of the adapter, making full use of the flexibility of the mobile terminal and reducing the cost of enterprise hardware wiring. As for the scenario of wine logistics supply chain tracking, the business engine processing module of the mobile middleware is needed to complete complex operations such as product warehousing, verification, and distribution and track the corresponding status.

The device access layer provides interfaces to various devices, interacts with hardware, provides a unified interface for different manufacturers of reading and writing devices and sensors, shields the heterogeneity of hardware, and facilitates hardware integration. Devices can include RFID fixed readers, handheld RFID readers, sensors, and RFID reading and writing modules integrated with mobile terminals. The data processing layer does preliminary processing of the data collected from the device access layer and has the characteristics of data collection, filtering, integration, and transmission. Different physical readers will be grouped by logical readers, and the collected RFID events will be integrated with the sensor events that arrive at the same time and filtered and regrouped according to the time stamp, RFID events, and sensing information. The business processing layer takes the encoded RFID events as input and uses its powerful business execution engine to track the business execution status and process the business execution logic. The execution engine separates the engine algorithm logic from the business execution logic and uses the enterprise service bus to distribute the execution services to different nodes to achieve a distributed service architecture. The application layer is the client of our middleware product, which is integrated with the middleware to achieve rapid development and deployment. Due to the use of wireless sensor networks and mobile terminals, our middleware not only supports traditional single-point RFID applications such as anticounterfeiting systems and access control systems but also extends the application scope to complex scenarios such as supply chain management and product quality monitoring.

\section{Analysis of Results}

4.1. Attitude Detection Analysis. Simulation analysis is done with MATLAB using different algorithms to derive the pose detection results of the pose detection samples in each segment. It is obvious from the figure that the support vector machine optimized by the genetic algorithm has the best results for pose detection, and the results of pose detection by SVM default parameters, SVM optimized by genetic algorithm and BP neural network algorithm is compared, it is found that the pose detection results of the support vector machine optimized by the genetic algorithm are closer to the actual values, and the pose detection accuracy is higher, as shown in Figure 3.

When the overturning action of the cargo occurs, as shown in Figure 4, the acceleration values in all three directions before the overturning are low, and the cargo is in a stable state, or there are low positive and negative alternating acceleration values, i.e., in a swaying state. When overturned, the acceleration of the goods in two or more directions changes sharply, and the maximum acceleration is related to the volume of the goods, the labeling position, and the direction of the overturning, but is much greater than the acceleration of the shaking and less than the acceleration generated by the direct impact. Cargo overturning is also often accompanied by the three-dimensional acceleration state law of the impact, that is, the overturning direction acceleration changes instantly, and fluctuations are obvious. The most important feature of the whole tipping action that distinguishes it from the impact is the existence of gravitational axial change before and after tipping, the change of attitude angle of $90^{\circ}$ in the time window before and after tips.

4.2. Performance Analysis of Positioning Algorithm. The performance trace of the corresponding localization algorithm for solving the problem, as computed by the localization algorithm described in the previous section, is shown in Figure 5. As can be seen from the algorithm performance tracking schematic, the objective value of the resulting transportation decision scheme gradually becomes better as the 


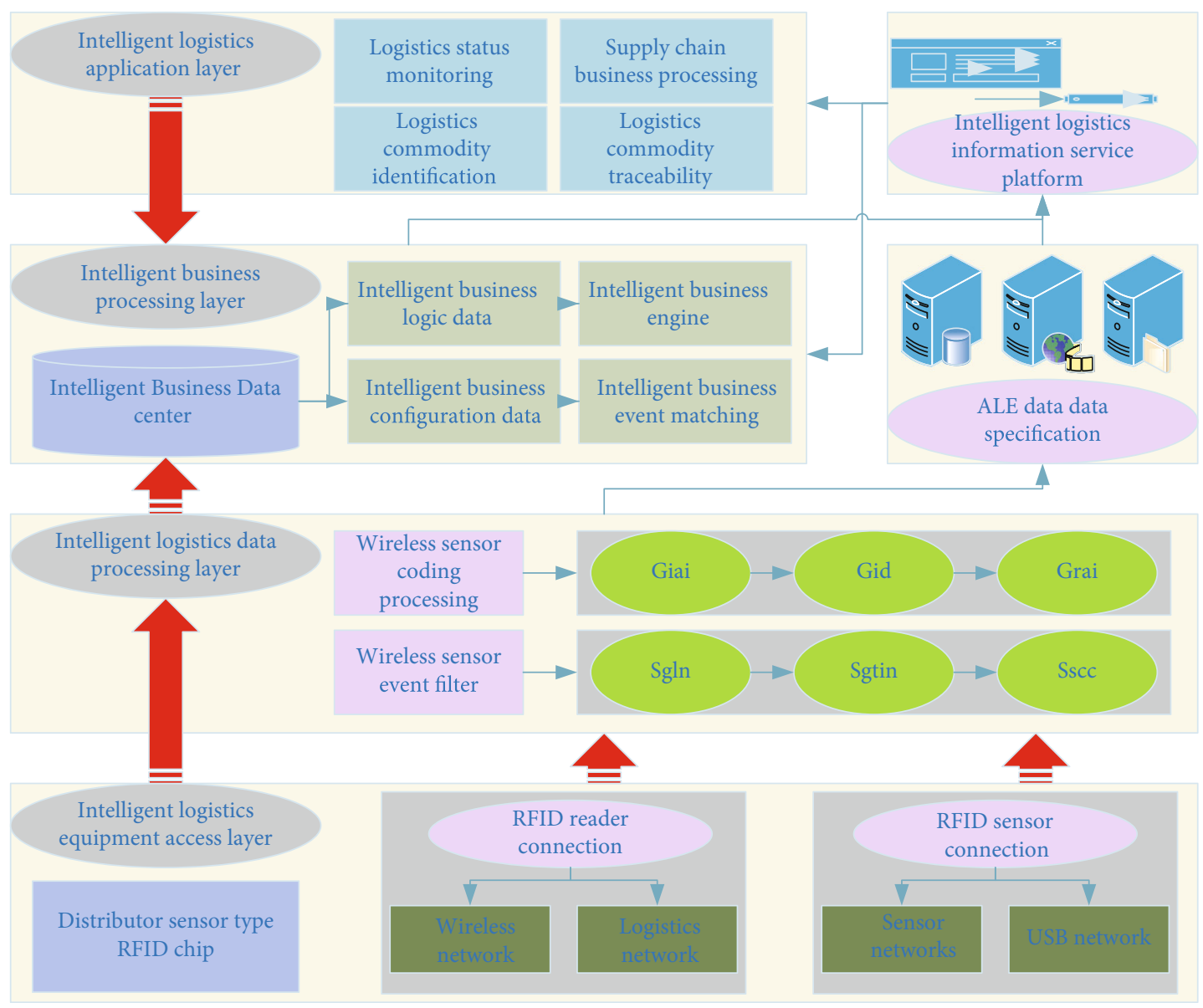

FIGURE 2: The overall architecture of intelligent logistics supply chain management system.

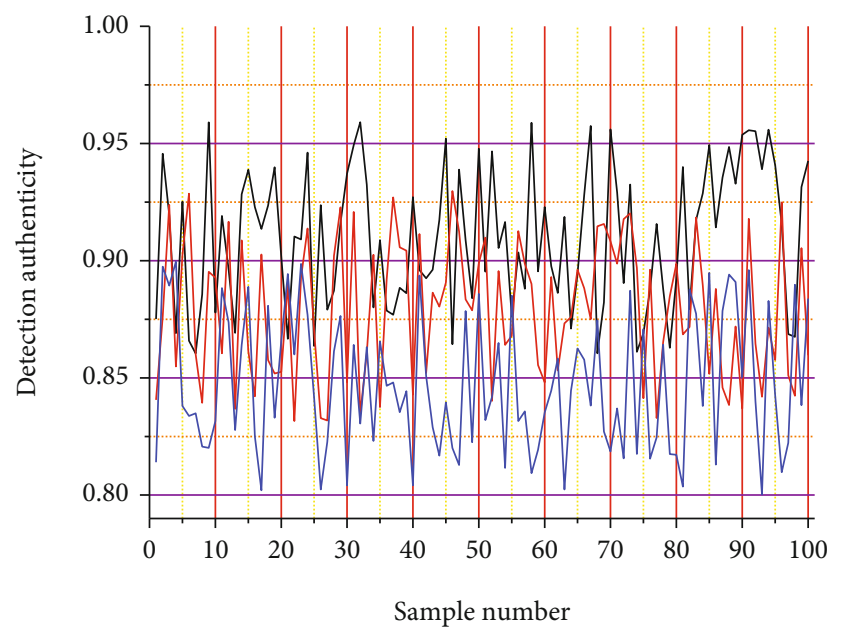

This article optimizes the algorithm
_ SVM optimization algorithm
_ BP neural network algorithm

FIgUre 3: Attitude detection test set.

number of evolutionary generations increases, and the algorithm converges to an optimized solution when it has evolved to about 300 generations.
Intelligent logistics in the storage and transportation process has a large hazardous and harmful nature, with transport path, transport time constraints, and dynamic nature. To effectively improve the efficiency of storage and transportation and optimize the control means, based on summarizing various influencing factors on transportation decision in intelligent logistics transportation network, under the constraints of section transportation capacity and transportation path time window, and to minimize social risk and transportation cost, the intelligent logistics transportation decision model is constructed to determine the transportation path, mode, and departure time of intelligent logistics between different ODs. Based on the characteristics of the model, the ant colony algorithm of elite strategy is designed to solve it. The transport decision calculation cases with 8 transit nodes and 6 OD flow directions show that the model and algorithm have good feasibility and effectiveness.

The localization error is observed for different values of $M$ under both localization algorithms, and it decreases gradually with the increase of the number of nodes and the increase of the localization coverage. The full localization coverage can be achieved only when the number of anchor nodes reaches 27 under the conventional localization algorithm; therefore, some nodes cannot be effectively localized when $M=15$. As shown in Figure 6(a), when the number 

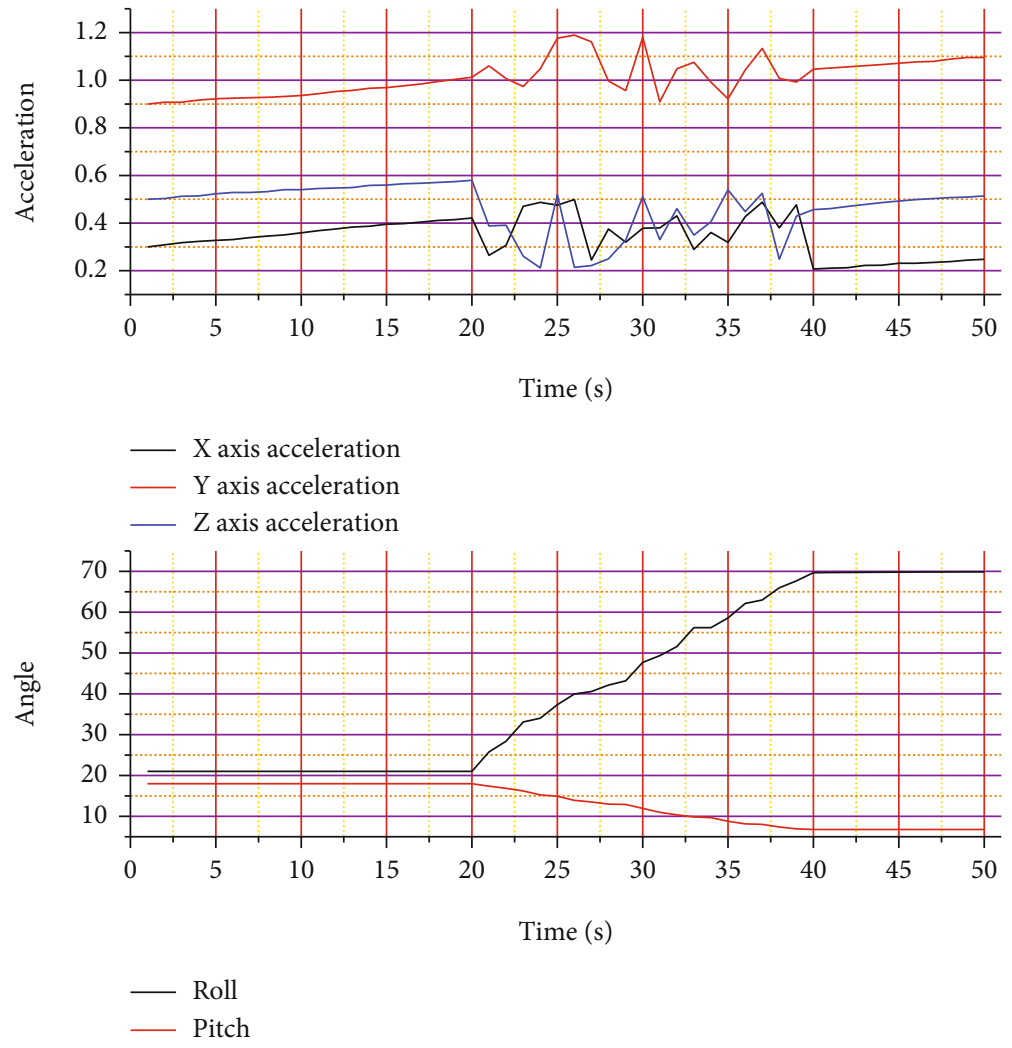

Figure 4: 3D acceleration and attitude angle characteristics in the overturned attitude.

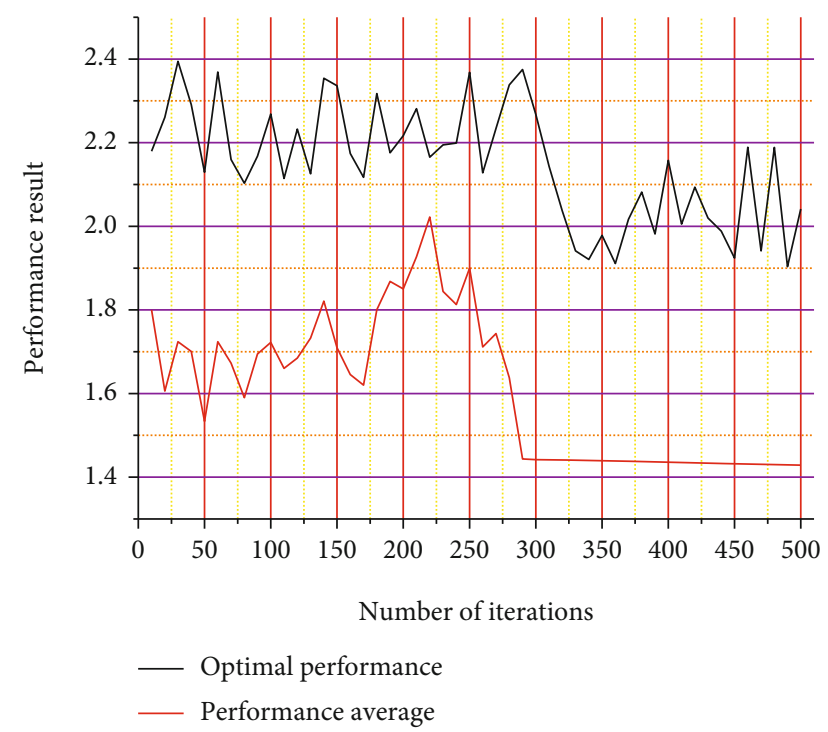

Figure 5: Performance tracking schematic.

of nodes $M=30$, as in Figure 6(b), all the tags can be localized. Figure 6(c) shows the error result of the two.

When the number of anchor nodes is small, the positioning cannot be completed at some locations, so the error is large. For the secondary localization algorithm designed in this paper, when the number of anchor nodes reaches 10 , the full coverage of the localization area has been completed, so that the error stabilizes in a small range. When the number of anchor nodes is large enough, the errors of both algorithms tend to be stable. The RSSI-based weighted centerof-mass algorithm and the proximity ranging based on moving anchor nodes in the secondary positioning method can further reduce the errors. The overall stability of the positioning system is further improved. The signal strengthbased weighted center-of-mass localization algorithm is used to compensate for the neglect of the anchor node credibility by the traditional algorithm. Through simulation experiments, it can be seen that the method can effectively reduce the number of nodes required for positioning under the same positioning area and provide a method to improve the effective coverage, and the positioning accuracy and coverage are better than the traditional triangular center-ofmass positioning algorithm and effectively reduce the cost of storage positioning.

4.3. System Simulation Analysis. The simulated data with the same RFID tag is repeatedly sent 100 times, and then the simulator generates a new tag number to send, while the middleware filter rules define that the same RFID data is received only once per second, so most of the data will be filtered out by the filter, and the remaining events are grouped, coded, and sent to the business processing layer. The business processing layer is a simple single-task linear process, and the system will test the response time and loss rate of the entire middleware when invoking the ESB component library service and the local service, respectively. The test results are shown in Figure 7. For the average response time 


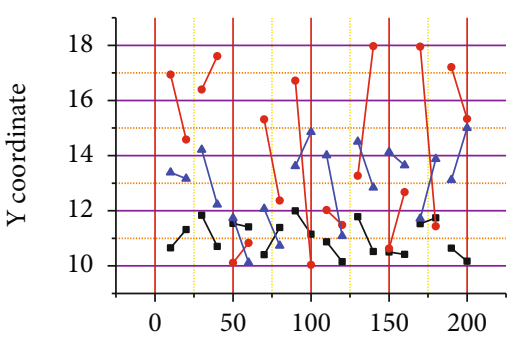

X coordinate

$\longrightarrow$ Anchor node position

$\longrightarrow$ Actual coordinate position

$\rightarrow$ Estimated coordinate position

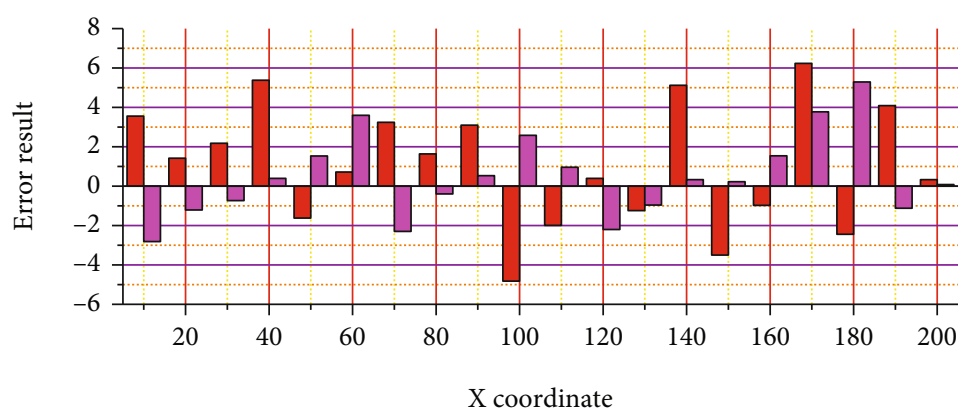

$M=15$ error distance

$\mathrm{M}=30$ error distance

FIgure 6: Positioning error of traditional positioning algorithm.

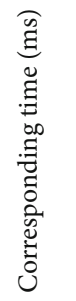

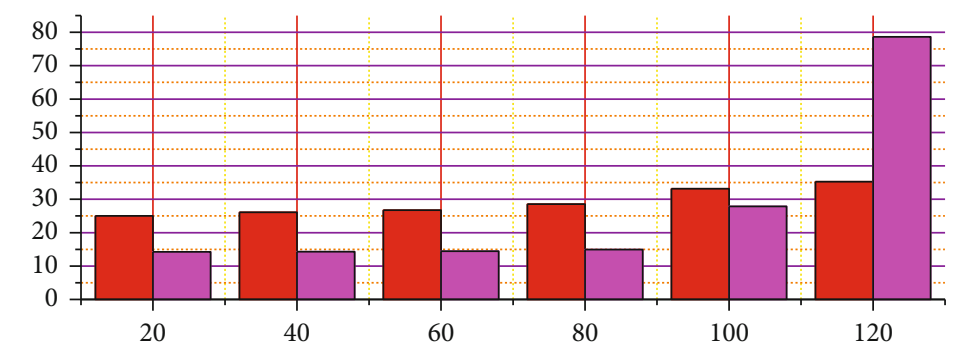

Test rate (bar/s)

Average response time (ESB)

Average response time (Local)

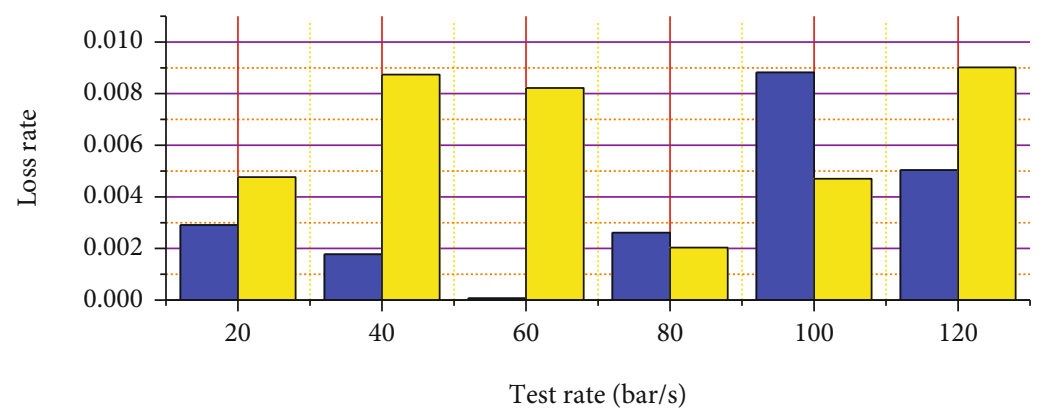

Loss rate (ESB)

Loss rate (Local)

Figure 7: RFID test result data. 


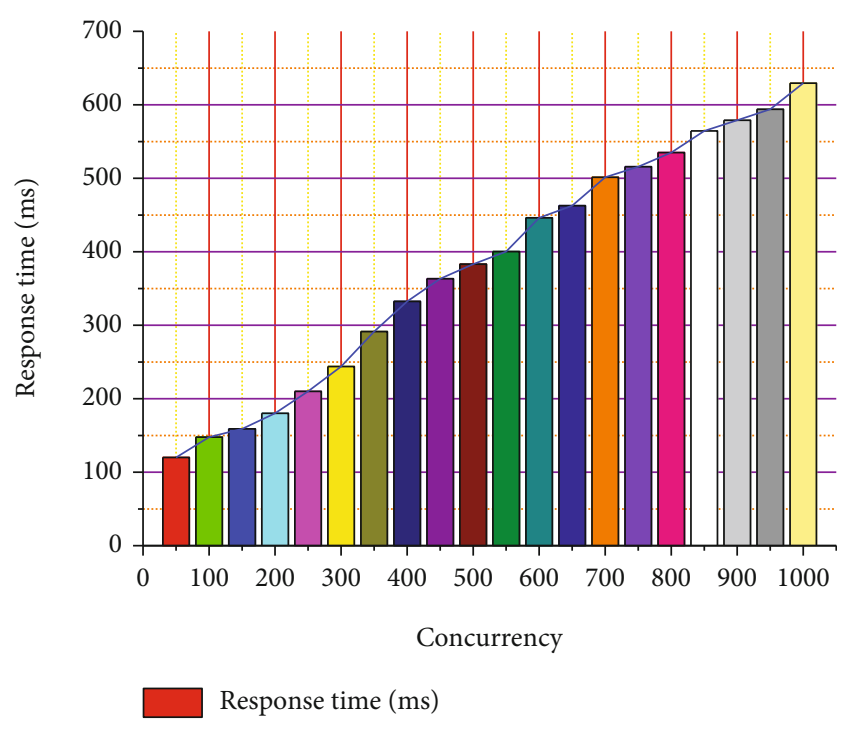

FIGURE 8: System response speed test.

(from the middleware receiving data to the end of processing), at lower test rates, the invocation of the ESB component library service takes longer than the direct invocation of the local component because of the additional network communication required; however, as the test rate increases, the average response time for the invocation of the ESB component library service only increases smoothly because the load is distributed among the ESB component's network services. However, the average response time for direct calls to local components increases dramatically as local resources become the bottleneck of the system. Therefore, for largescale data concurrent network environments, using a distributed ESB component library can greatly improve the scalability and performance of the system. 100,000 test events were sent continuously at a rate of 100 events per second, and the test results showed that the server was running normally, with an average response time of $26.316 \mathrm{~ms}$ and a system loss rate of $0.000218(218 / 100,000)$.

For the intelligent logistics supply chain management system, the number of concurrent processes is one of the key indicators reflecting the speed of mass sending; therefore, in the process of software testing, the test for the number of sending concurrency is most important. For this indicator, in this paper, a stress test was conducted on the system by sending a large number of tag updates in a short period and using a cyclic sending method to observe the performance load capacity and information processing capacity of the system. At the same time, the content and number of tags sent are recorded to see if there are any problems such as omissions at the receiving end and to check the integrity of the system sends. Through the test, the system latency increased significantly when the system concurrency was at 600 , and the sending performance of the system became significantly slower. The system latency increased significantly, and the statistics of sending-receiving data showed that the system began to lose packets at 700 concurrencies. The experimental results are shown in Figure 8. From the above experimental data, it can be seen that the range of parallel data that the system can accept for normal operation is $(1000,1500)$, and if the parallel data that the system needs to process exceeds this range, its operation speed will be rapidly reduced. At this stage, the system capacity can meet the actual demand.

The logistics company will need to check the material and label information again when the material is loaded and recover the remaining RFID label in the hands of the material supplier, to clarify the responsibility of all parties. After using RFID reader to check the relevant information, by the requirements of the ministry in charge of materials, fill out the Excel material information statistics table and label information statistics table, and through the form of mail will be timely information statistics table back to the ministry in charge of power grid materials, by the management personnel in charge of the ministry in charge of the Excel table of information into the materials management information system, to let all parties in the engineering supply chain timely understand the current status of the tags. After arriving at the designated unloading place, the intelligent logistics supply chain needs to use an RFID reader to count the materials and label condition for the last time, fill in the information statistics table according to the form automatically generated by the reader and the requirements of the material competent department mouth in time, and pass back through the form of mail-in time, and the management personnel of the material competent department mouth will import the information of the Excel table into the material management information system, to let the personnel of the project supply chain let all parties in the engineering supply chain understand the existing status of the label in time.

\section{Conclusion}

The complementary characteristics of wireless sensor networks and RFID technology make the combination of the two a great opportunity. This new fusion structure also provides new ideas to solve some key problems in warehousing logistics. In this paper, based on the in-depth study of the above two technologies, we propose solutions to the urgent problems of warehouse positioning and in-transit cargo security in the logistics process. And on this basis, it broadens the whole logistics process of warehousing and distribution and designs a monitoring system framework to realize the management process of the main business of warehousing and logistics. For the traditional RFID-based warehouse positioning method hardware wiring complex, the number of positioning nodes is too large, the effective coverage rate is low, and this paper designs a WSN and RFID integration of the anchor node; first, the node's wireless data transmission capabilities can directly and effectively solve the problem of wiring complexity. Second, the positioning anchor node without data line bondage can adopt a mobile strategy, which also provides an opportunity to seek to reduce the number of positioning nodes. In this paper, we define and classify the abnormal behavioral pose of cargo, use plus six-axis velocity sensors to obtain 3D acceleration data and pose angle of cargo in transit, and 
provide an algorithm for detecting behavioral pose. The method effectively solves the problem of redundant processing of cargo under normal conditions, provides time detection window division, and extracts key data segments; through the analysis of statistical features of a large amount of experimental data, an effective feature vector is designed for key data segments to establish a decision tree classification method, and the experiment proves that the method has high classification accuracy and can effectively solve the problem of monitoring the behavioral attitude of cargo in transit. In the implementation of this paper, although the problem of anticheating is also considered, there are still many shortcomings, and the common cheating and invasion methods can be better prevented, but for professional invasion and cheating, the system still has many shortcomings. And the research on the collision problem of tags is not very deep. This is where the subsequent research can go further.

\section{Data Availability}

The data used to support the findings of this study are available from the corresponding author upon request.

\section{Conflicts of Interest}

The authors declare that they have no known competing financial interests or personal relationships that could have appeared to influence the work reported in this paper.

\section{References}

[1] K. Leng, L. Jin, W. Shi, and I. van Nieuwenhuyse, "Research on agricultural products supply chain inspection system based on internet of things," Cluster Computing, vol. 22, no. S4, pp. 8919-8927, 2019.

[2] Q. Gao, S. Guo, X. Liu, G. Manogaran, N. Chilamkurti, and S. Kadry, "Simulation analysis of supply chain risk management system based on IoT information platform," Enterprise Information Systems, vol. 14, no. 9-10, pp. 1354-1378, 2020.

[3] X. Yan and J. Li, “Animal intelligent logistics management based on RFID technology," Revista Científica de la Facultad de Ciencias Veterinarias, vol. 29, no. 6, pp. 1772-1781, 2019.

[4] Y. Ding, M. Jin, S. Li, and D. Feng, "Smart logistics based on the internet of things technology: an overview," International Journal of Logistics Research and Applications, vol. 24, no. 4, pp. 323-345, 2021.

[5] N. Lei, "Intelligent logistics scheduling model and algorithm based on internet of things technology," Alexandria Engineering Journal, vol. 61, no. 1, pp. 893-903, 2022.

[6] C. Çeken and D. Abdurahman, "Simulation modeling of an iot based cold chain logistics management system," Sakarya University Journal of Computer and Information Sciences, vol. 2, no. 2, pp. 89-100, 2019.

[7] M. Ben-Daya, E. Hassini, and Z. Bahroun, "Internet of things and supply chain management: a literature review," International Journal of Production Research, vol. 57, no. 15-16, pp. 4719-4742, 2019.

[8] A. P. Pandian, "Artificial intelligence application in smart warehousing environment for automated logistics," Journal of Artificial Intelligence, vol. 1, no. 2, pp. 63-72, 2019.
[9] C. Y. Lam and W. H. Ip, "An integrated logistics routing and scheduling network model with RFID-GPS data for supply chain management," Wireless Personal Communications, vol. 105, no. 3, pp. 803-817, 2019.

[10] M. Abdirad and K. Krishnan, "Industry 4.0 in logistics and supply chain management: a systematic literature review," Engineering Management Journal, vol. 33, no. 3, pp. 187-201, 2021.

[11] M. A. M. Sadeeq and S. Zeebaree, "Energy management for internet of things via distributed systems," Journal of Applied Science and Technology Trends, vol. 2, no. 2, pp. 59-71, 2021.

[12] Q. Zhou, Z. Zhang, and Y. Wang, "Research on safety management system optimization of B2C E-commerce intelligent logistics information system based on data cube," Journal of Intelligent \& Fuzzy Systems, vol. 38, no. 2, pp. 1585-1592, 2020.

[13] H. Ping, J. Wang, Z. Ma, and Y. Du, "Mini-review of application of IoT technology in monitoring agricultural products quality and safety," International Journal of Agricultural and Biological Engineering, vol. 11, no. 5, pp. 35-45, 2018.

[14] C. K. M. Lee, Y. Lv, K. K. H. Ng, W. Ho, and K. L. Choy, "Design and application of internet of things-based warehouse management system for smart logistics," International Journal of Production Research, vol. 56, no. 8, pp. 2753-2768, 2018.

[15] M. Attaran, "Digital technology enablers and their implications for supply chain management supply chain forum: an international journal," Taylor \& Francis, vol. 21, no. 3, pp. 158-172, 2020.

[16] L. W. Wong, G. W. H. Tan, V. H. Lee, K. B. Ooi, and A. Sohal, "Unearthing the determinants of Blockchain adoption in supply chain management," International Journal of Production Research, vol. 58, no. 7, pp. 2100-2123, 2020.

[17] X. Hu, L. Sun, Y. Zhou, and J. Ruan, "Review of operational management in intelligent agriculture based on the internet of things," Frontiers of Engineering Management, vol. 7, no. 3, pp. 309-322, 2020.

[18] S. Paul, A. Chatterjee, and D. Guha, "Study of smart inventory management system based on the internet of things (IOT)," International Journal on Recent Trends in Business and Tourism (IJRTBT), vol. 3, no. 3, pp. 27-34, 2019.

[19] Y. Mao and L. Zhang, "Design and implementation of port bulk storage management system based on internet of things technology," Journal of Coastal Research, vol. 98, no. sp1, pp. 62-66, 2019.

[20] A. I. Vlasov, P. V. Grigoriev, A. I. Krivoshein, V. A. Shakhnov, S. S. Filin, and V. S. Migalin, "Smart management of technologies: predictive maintenance of industrial equipment using wireless sensor networks," Entrepreneurship and Sustainability Issues, vol. 6, no. 2, pp. 489-502, 2018. 